\title{
Caring about what happens to people with COPD
}

\section{Nicholas S Hopkinson}

Even in an adequately resourced system, admission to hospital with an acute exacerbation of COPD (AECOPD) could be a frightening and unpleasant experience for patients with the condition and for their relatives. Unfortunately, the UK Government has made a political choice to underfund the National Health Service (NHS) by more than $£ 20$ billion per year by 2020 and there has already been a $26 \%$ cut in the number of people over 65 receiving local authority social care since 2010. ${ }^{1}$ The latest report from the UK's 2014 National COPD audit programmeCOPD: who cares when it matters most? ${ }^{2}$ warns of patients 'on a continuous cycle of admission' with an 'overriding impression provided by the data of a system that is not only stressed, but is ultimately failing COPD patients'. The report found that $8 \%$ of patients discharged following an AECOPD died within 90 days of admission, 25\% were readmitted within 30 days and $43 \%$ within 90 days. Fewer than half $(43 \%)$ of the readmissions were due to COPD. The number of prior admissions to hospital was associated with postdischarge mortality and subsequent readmission risk. Readmission was much more likely in those with a longer length of stay and in patients who had been discharged to sheltered accommodation.

In Thorax, Echevarria and colleagues describe the development of a new prognostic tool for use in patients admitted with an AECOPD. ${ }^{3}$ The initial derivation and subsequent validation cohorts, including six UK centres, were those that were also used in the development of the Dyspnoea, Eosinopenia, Consolidation, Acidaemia, and atrial Fibrillation (DECAF) score, a tool to identify patients who can be safely discharged. ${ }^{45}$ Patient characteristics, selected on the basis of literature review and clinical plausibility, and which were consistently recorded across sites, were related to the risk of readmission or death within 90 days of discharge. The outcome of this is the PEARL score, which includes Prior admission history, Extended medical research council (MRC) dyspnoea score

Correspondence to Dr Nicholas S Hopkinson, NIHR Respiratory Disease, Biomedical Research Unit at the Royal Brompton and Harefield NHS Foundation Trust and Imperial College London, London SW3 6NP, UK; n.hopkinson@ic.ac.uk
(eMRCD), Age over 80, Right ventricular failure and Left Ventricular failure. The eMRCD is a development of the conventional MRC dyspnoea score which includes modification of the category 5 to 'inability to leave the house without assistance'; a measure of frailty (5b-'the inability to wash and dress independently'); and clearer transition between levels. The score ranges from 0-9 with low (0-1), medium (2-4) and high (5-9) risk groups identified. At 30 days, readmission or death occurred in $11 \%, 24 \%$ and $40 \%$, respectively. The performance of the PEARL score was compared with other predictive scores (age dyspnoea obstruction index (ADO) ${ }^{6}$ DECAF, eMRCD ${ }^{4}{ }^{5}$ BODEX $^{7}$ CODEX $^{8}$ dyspnoea, obstruction, smoking, exacerbations index $(\mathrm{DOSE})^{9}$ and $\mathrm{LACE}^{10}$ ). The area under the receiver-operating characteristic curve (AUROC) for PEARL in the validation cohort was 0.7 , superior to all other prognostic scores tested.

The authors conclude that the PEARL score is a robust and consistent predictor of death and readmission following AECOPD, that it is superior to other tools and that it could be used to aid clinical decision making and resource allocation. These three claims, broadly validity, superiority and utility, deserve further consideration.

The context for the development of the PEARL score is sound: an initial derivation cohort and then reproduction in a validation cohort. The hospitals involved were chosen to ensure variations in populations and clinical practice and pathways were covered and patient acquisition seems robust, with few excluded from the study, mostly for reasons of malignancy. The 90-day readmission rate in the study population was similar to that observed in the 2014 Royal College of Physicians audit ${ }^{2}$ and other predictive scores evaluated in this study had similar performance characteristics to those seen when they have been evaluated previously. It is likely therefore that the results are generalisable in the sense that the same parameters would likely yield the same results across the NHS. There are some issues regarding the choice of these parameters. The McNamara fallacy warns of the danger of focusing on what is easily measured. Clinical measurements are made and recorded for a range of reasons-well-established usefulness for some, but also tradition and ease of documentation. A general risk with PEARL, and indeed other scores, is that being based on what is available the score may entrench inadequate data collection or clinical assessment. We should retain the possibility that other, perhaps novel, parameters might predict outcomes better and would therefore become worth the additional effort to make and record them systematically. The assessment of left ventricular failure was based on preadmission diagnosis confirmed by echocardiography, right heart failure on the basis of a clinical diagnosis of cor pulmonale including at the time of admission. This is a practical approach, though it is partly dependent on prior investigation history. Other definitions linked, for example, to medications patients were taking or specific biomarkers might have yielded different results and this should be considered for future investigation.

Is PEARL superior to other scores? The data required should be easy to collect and the AUROC data suggest that for the purpose of predicting death or readmission post AECOPD it is more accurate, with higher values than all other comparators both in the derivation and validation cohorts. However, the numerical differences were small and an AUROC of 0.7 is not itself a particularly high value for a predictive test. Of note, the AUROC curve for the eMRCD alone for 90-day readmission was 0.61 in the external validation cohort, better than ADO and DOSE.

Zweig and Campbell discussing ROC curves note that 'diagnostic accuracy refers to the quality of the information provided by the classification device and should be distinguished from the usefulness, or actual practical value, of the information'. ${ }^{11}$ So, how useful is the score? It could be helpful for stratification in trials designed to evaluate interventions intended to improve short or longer term post AECOPD outcomes. It could also improve the validity of data in audits involving readmission, as it allows a more satisfactory adjustment for case mix both for internal assessment and comparison between providers.

More problematic is use 'to aid clinical decision making and resource allocation'. Prior admission history and age are not modifiable risk factors. Multimorbidity is the norm rather than the exception in COPD $-50 \%$ of patients with COPD have three or more other long-term conditions $^{12}$ and frailty is a common feature of people with the disease. ${ }^{13}$ The PEARL score does reinforce frailty as an important predictor of poor outcomes in patients with COPD. ${ }^{13} 14$ Postexacerbation pulmonary rehabilitation is an effective intervention to reduce readmission risk ${ }^{15}{ }^{16}$ which 
Table 1 The Breathing SPACE approach to breathless conditions developed by The London Respiratory Network ${ }^{18}$

\begin{tabular}{ll}
\hline Smoking & Offer evidence-based smoking cessation support for all \\
Pulmonary disease & Offer prompt spirometry \\
& Prioritise high-value care \\
Anxiety & Identify and support psychosocial problems \\
Cardiac disease & Dual diagnoses are common \\
& Do not undertreat \\
Exercise & Pulmonary rehabilitation \\
& Encourage physical activity \\
\hline SPACE, Smoking, Pulmonary disease, Anxiety, Cardiac disease, Exercise.
\end{tabular}

addresses frailty, but it is indicated for any patient following admission with an AECOPD, so the score is not contributory as such. The frailest patients will be likely to face the greatest barriers to participation. There may be a role for technological adjuncts such as telecoaching ${ }^{17}$ to improve activity levels, but this is unlikely to address the need that frail individuals have for face-to-face interaction and support.

The fact that the presence of known cardiac disease predicted death and readmission underscores the importance of these comorbidities in COPD, as highlighted recently in the Breathing SPACE ${ }^{18}$ (Smoking, Pulmonary disease, Anxiety, Cardiac disease, Exercise) approach for any breathless condition proposed by the London Respiratory Network (table 1). There are certainly data to suggest that cardiac disease is systematically undertreated in patients with $\mathrm{COPD}^{18}$ so it may be that application of the score will be a useful nudge to address this. The COPD discharge care bundle was developed out of the limited evidence base available for interventions to improve outcomes post-AECOPD. ${ }^{19}$ A 'consider and optimise cardiac care' item may be helpful. Importantly, the score only addresses known cardiac disease-the optimum diagnostic strategy to identify cardiac comorbidity in COPD remains to be defined.

Across the world, while demands placed by AECOPD on health systems are increasing, ${ }^{20-22}$ COPD is at the cutting edge of the social determinants of health. ${ }^{23}$ Prevalence and mortality rates are strongly associated with social class and COPD has roots in prenatal and childhood disadvantage, as well as steep social gradients in smoking rates and occupational exposures to dust, fumes and chemicals. Smoking and hence COPD rates are extremely high in people who are homeless and those who have mental health conditions, while rising fuel poverty has meant that many patients with COPD live in cold damp homes. Provision of pulmonary rehabilitation, one of the highest value care items for patients with COPD, remains inadequate.
There has historically been less advocacy by and for patients with the condition than for other conditions with a similar disease burden such as coronary disease and common malignancies. ${ }^{24}$ In terms of resource allocation, even the 'low-risk' PEARL score group still had a substantial 90-day death or readmission if it were effective, it would be reasonable to deny this group because that rate was too low. Certainly not pulmonary rehabilitation, or optimisation of cardiac treatment, or action to address social care needs.

Reducing hospital readmission is an important objective for both individuals and health and social care systems more broadly, although expectations of the extent to which this can be achieved need to be realistic, especially in the absence of the necessary health and social care resources. ${ }^{1}$ From a healthcare perspective, the PEARL score data add to the picture which is emerging of the importance, in AECOPD, of combining optimum medical intervention which focuses on both lung and cardiac disease, with recognition and action to identify and deserve this.

Twitter Follow Nicholas Hopkinson @COPDdoc

Contributors NSH wrote this editorial.

Competing interests None declared.

Provenance and peer review Commissioned; externally peer reviewed.

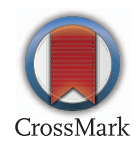

To cite Hopkinson NS. Thorax 2017;72:683-685.

Published Online First 2 May 2017

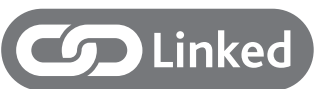

- http://dx.doi.org/10.1136/thoraxjnl-2016-209298

Thorax 2017;72:683-685.

doi:10.1136/thoraxjnl-2017-210065 rate of $21 \%$. It is not clear what resource, address frailty. All patients with COPD

\section{REFERENCES}

1 The Kings Fund, The Health Foundation, The Nuffield Trust. The Autumn Statement: joint statement on health and social care. 2016. https://www.kingsfund. org.uk/publications/autumn-statement-2016

2 Royal College of Physicians. National COPD Audit Programme. COPD: Who care when it matters most? 2017. https://www.rcplondon.ac.uk/ projects/outputs/copd-who-cares-when-it-mattersmost-outcomes-report-2014

3 Echevarria C, Steer J, Heslop-Marshall K, et al. The PEARL score predicts 90-day readmission or death after hospitalisation for acute exacerbation of COPD. Thorax 2017;72:686-93.

4 Steer J, Gibson J, Bourke SC. The DECAF Score: predicting hospital mortality in exacerbations of chronic obstructive pulmonary disease. Thorax 2012;67:970-6.

5 Echevarria C, Steer J, Heslop-Marshall K, et al. Validation of the DECAF score to predict hospital mortality in acute exacerbations of COPD. Thorax 2016;71:133-40.

6 Puhan MA, Garcia-Aymerich J, Frey M, et al. Expansion of the prognostic assessment of patients with chronic obstructive pulmonary disease: the updated BODE index and the ADO index. Lancet 2009;374:704-11.

7 Soler-Cataluna JJ, Martinez-Garcia MA, Sanchez LS, et al. Severe exacerbations and BODE index: two independent risk factors for death in male COPD patients. Respir Med 2009;103:692-9.

8 Almagro P, Soriano JB, Cabrera FJ, et al. Short- and medium-term prognosis in patients hospitalized for COPD exacerbation: the CODEX index. Chest 2014; 145:972-80.

9 Jones RC, Donaldson GC, Chavannes NH, et al. Derivation and validation of a composite index of severity in chronic obstructive pulmonary disease: The DOSE Index. Am J Respir Crit Care Med 2009;180:1189-95.

10 van Walraven C, Dhalla IA, Bell C, et al. Derivation and validation of an index to predict early death or unplanned readmission after discharge from hospital to the community. CMAJ 2010;182:551-7.

11 Zweig MH, Campbell G. Receiver-operating characteristic (ROC) plots: a fundamental evaluation tool in clinical medicine. Clin Chem 1993;39:561-77.

12 Barnett K, Mercer SW, Norbury M, et al. Epidemiology of multimorbidity and implications for health care, research, and medical education: a cross-sectional study. Lancet 2012;380:37-43.

13 Patel MS, Mohan D, Andersson YM, et al. Phenotypic characteristics associated with reduced short physical performance battery score in COPD. Chest 2014;145:1016-24.

14 Kon SSC, Jones SE, Schofield SJ, et al. Gait speed and readmission following hospitalisation for acute exacerbations of COPD: a prospective study. Thorax 2015;70:1131-7.

15 Seymour JM, Moore L, Jolley CJ, et al. Outpatient pulmonary rehabilitation following acute exacerbations of COPD. Thorax 2010;65:423-8.

16 Puhan MA, Gimeno-Santos E, Scharplatz M, et al. Pulmonary rehabilitation following exacerbations of chronic obstructive pulmonary disease. Cochrane Database Syst Rev 2011;(10): Cd005305.

17 Demeyer $H$, Louvaris Z, Frei $A$, et al. Physical activity is increased by a 12 -week semiautomated telecoaching programme in patients with COPD: a multicentre randomised controlled trial. Thorax 2017;2017;72:415-23.

18 Hopkinson NS, Baxter N. Breathing SPACE-a practical approach to the breathless patient. NPJ Prim Care Respir Med 2017;27:5. 
19 Hopkinson NS, Englebretsen C, Cooley N, et al. Designing and implementing a COPD discharge care bundle. Thorax 2012;67:90-2.

20 Molinari N, Chanez P, Roche N, et al. Rising total costs and mortality rates associated with admissions due to COPD exacerbations. Respir Res 2016;17:14.

21 Khakban A, Sin DD, FitzGerald JM, et al. The projected epidemic of chronic obstructive pulmonary disease hospitalizations over the next 15 years. A population-based perspective. Am J Respir Crit Care Med 2017;195:287-91.

22 Royal College of Physicians. COPD Who cares matters: Full Report National Chronic Obstructive Pulmonary Disease (COPD) Audit Programme. Clinica audit of COPD exacerbations admitted to acute units in England and Wales 2014. London: Royal College of Physicians, 2015.
23 Prescott E, Vestbo J. Socioeconomic status and chronic obstructive pulmonary disease. Thorax 1999;54:737-41.

24 Hopkinson NS. What is breathing worth? The economic cost of lung disease. The BMJ Opinion [blog], 4 Apr 2017. http://blogs.bmj.com/bmj/2017/ 04/04/nick-hopkinson-what-is-breathing-worth-theeconomic-cost-of-lung-disease/ 